\title{
Proteasomes, caught in the act
}

\author{
Cell Research (2017) 27:307-308. doi:10.1038/cr.2017.22; published online 21 February 2017
}

\begin{abstract}
Although energy-dependent protein destruction by the proteasome has been known for over 30 years, how this intricate molecular machine uses ATP to power protein degradation has remained very poorly understood. In a recently published paper, Ding et al. present a snapshot of the proteasome mid-catalysis, yielding new and unexpected insights into the catalytic mechanism of this ATPpowered multisubunit machine.
\end{abstract}

Cells rely on large multisubunit molecular machines to conduct many complex biological processes, such as protein synthesis, folding, and degradation. In eukaryotes, most regulated protein degradation is conducted by the proteasome, a massive 66-subunit ATP-dependent protease complex. Although recent advances in cryo-electron microscopy (cryo-EM) have yielded an onslaught of information about the proteasome, none have yet revealed how ATP drives the proteasome's catalytic cycle. By pharmacologically trapping the proteasome in a transition state, Ding et al. [1] provide a snapshot of the proteasome's molecular motor in action. This snapshot yields answers as well as poses new questions regarding the catalytic mechanism of this fascinating multisubunit machine.

The proteasome consists of three subcomplexes (Figure 1A), the barrelshaped core particle (CP), which houses the peptidase active sites; the ringshaped regulatory particle (RP) base, which abuts the ends of the CP; and the RP lid, which embraces the base and $\mathrm{CP}$ and hovers over their central pores. These subcomplexes function together much like an assembly line to bind the substrate protein, unfold it, remove its proteasomal targeting signal, and to cleave it into short peptides. Like the macroscopic machines we encounter in everyday life, the proteasome contains numerous moving parts and depends on a motor to power and coordinate its multiple activities. The motor of the proteasome is a ring of six AAA+ family ATPase subunits within the base. These six ATPases, Rpt1-6, undergo interconnected conformational changes upon ATP binding and hydrolysis. These conformational changes serve two critical functions during substrate degradation. First, they reposition the lid, base, and CP subcomplexes relative to one another, providing a clear path for the substrate through the complex [2, 3]. Second, they cause the ATPases to grasp and pull downward on unstructured parts of the substrate via conserved, paddle-like loops that point into the pore of the ATPase ring (Figure 1B). This pulling mechanically unfolds the substrate, and feeds it into the CP active sites.

Although numerous cryo-EM structures of the proteasome have recently been published, in all cases this complex appears to be in a "dwell" phase, in which it is essentially sitting still between mechanical movements and ATP hydrolysis events. To capture a view of the proteasome mid-catalysis, Ding et al. determined the structure of the proteasome in the presence of the ATP analog ADP-AlF . In other ATPases [4, 5], provision of ADP-AlF locks the ATPase motor in a transitionlike state of the ATP hydrolysis cycle, and thus, their structure likely reveals the proteasome's ATPase motor mid- stroke. In their model, the ATPase ring of the proteasome is in a very different conformation than that observed in all previous dwell-phase structures, and provides new insights into the nature of the ATPase cycle and its relation to substrate unfolding.

Ding et al. compared their structure produced in the presence of ADP-AlF with a second structure produced in the presence of ATP, which expectedly adopted the dwell-phase conformation. Two major differences between these structures were immediately evident. First, in the dwell-phase model, there was evidence of density consistent with nucleotide in each of the six ATPases' active sites (Figure 1B). Although in agreement with recent structures from other groups $[6,7]$, this is somewhat surprising because the proteasome and other related hexameric AAA+ ATPases are thought to bind at most four nucleotides simultaneously. As cryo-EM depends on averaging together densities from tens or hundreds of thousands of individual particles, this may simply represent an average of all discrete nucleotide configurations present in the sample, and would imply that different nucleotide arrangements are possible in the dwell conformation.

In stark contrast to the resting state structure, nucleotide can be readily modeled into density in the active sites of four consecutive ATPase subunits, Rpt1 - Rpt5 - Rpt4 - Rpt3, in the ADP$\mathrm{AlF}_{\mathrm{x}}$ structure, with subunits Rpt2 and Rpt6 appearing empty. Such a configuration is consistent with a proposed rotary hydrolysis mechanism [8, 9] in which ATP hydrolysis takes place in coordinated waves counterclockwise 
A

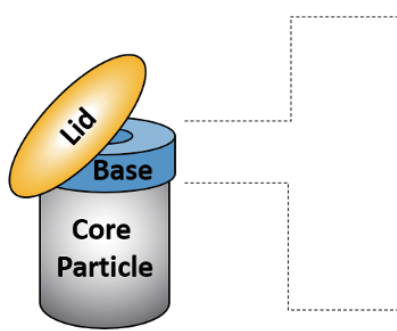

B

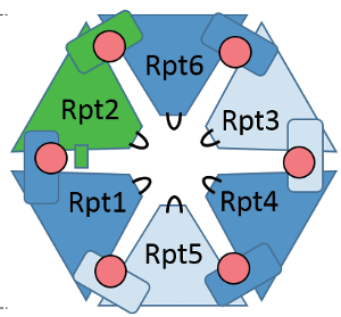

ATP (dwell)
C

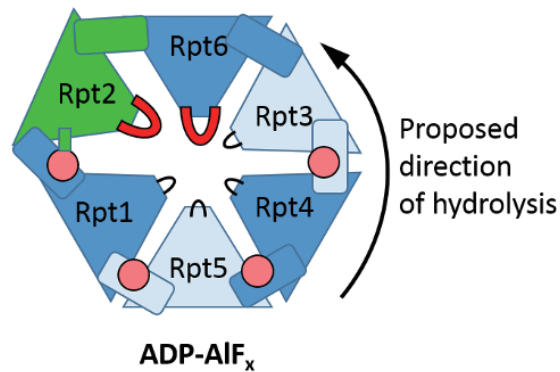

Figure 1 (A) The proteasome consists of lid, base, and core particle (CP) subcomplexes. (B) The base contains a hexameric ring of six ATPase subunits, Rpt1-6. Each subunit consists of a trapezoidal large domain and a rectangular small domain. The interface between the large and small domains forms the nucleotide-binding pocket. Density for nucleotide (red circle) is present in each pocket. The pore loops of each subunit point toward the ATPase ring pore, and the arginine fingers of the clockwise adjacent subunit (rectangle; only the Rpt2 arginine fingers are depicted) point toward the neighboring nucleotide-binding pocket. In the dwell state shown here, the pore loops of Rpt2 and Rpt6 are in low positions, and the Rpt2 arginine finger is not contacting nucleotide in the Rpt1 binding pocket. (C) In the ADP-AIF structure, Rpt1, 5, 3, and 4 display bound nucleotide, whereas Rpt2 and Rpt6 are empty. Rpt2 has undergone a large rotation that decreases its contact with Rpt6 or Rpt1. This rotation raises the Rpt2 and Rpt6 pore loops (large red loops), and positions the Rpt2 arginine fingers into the catalytic pocket of Rpt1.

around the ring. However, it is at odds with nucleotide binding configurations known for other AAA+ family ATPases that unfold proteins, such as the bacterial ClpX [10]. This suggests that the catalytic cycle of the proteasome may diverge from that of related ATPases.

The second, and perhaps most striking feature of the ADP-AlF $F_{x}$ model, is that the Rpt2 subunit has undergone an $\sim 38^{\circ}$ rotation up and away from the center of the ATPase ring compared to the dwell state, greatly reducing its contact with neighbors Rpt1 and Rpt6. This positions the pore loops of Rpt2 and Rpt6 much higher than in dwell state proteasome structures (Figure 1C). This elevated position of the Rpt 2 and Rpt6 pore loops is ideal for grasping the substrate near the entrance to the pore for unfolding and translocation into the CP. Thus, elevation of these pore loops may represent either a "resetting" of Rpt2 or Rpt6 after firing and release of nucleotide, or instead a priming event preceding their next round of substrate pulling.

A second important outcome of the Rpt2 rotation is that it positions key
Rpt2 side chains, called arginine fingers, into the Rpt1 nucleotide-binding pocket. Arginine fingers are known to sense nucleotide bound by adjacent subunits in the ATPase ring [11], but the arrangement of nucleotide counterclockwise to Rpt 2 raises the intriguing possibility that they could contribute directly to catalysis [9]. Namely, protrusion of the Rpt2 arginine fingers into the Rpt1 nucleotide pocket may be necessary to stimulate ATP binding or hydrolysis by Rpt1, implicating Rpt1 as the initiator of ATP hydrolysis around the ring [8]. Consistent with such a mechanism, no distention similar to that for Rpt2 was observed for any of the other five ATPases. Although the possibility that this conformation simply represents a lowest energy state or a metastable hydrolysis step with a particularly high energy of activation cannot yet be excluded, the model reported by Ding et al. provides one of the first fascinating glimpses into the proteasome in motion, and will undoubtedly inspire follow-up analyses to better understand coordination of processing events in this complicated macromolecular machine.

\section{Robert J Tomko Jri}

${ }^{1}$ Department of Biomedical Sciences, Florida State University College of Medicine, $1115 \mathrm{~W}$. Call Street, Tallahassee, FL 32306, USA

Correspondence: Robert J Tomko Jr

E-mail: robert.tomko@med.fsu.edu

\section{References}

1 Ding Z, Fu Z, Xu C, et al. Cell Res 2017 Jan 20. doi:10.1038/cr.2017.12

2 Matyskiela ME, Lander GC, Martin A. Nat Struct Mol Biol 2013; 20:781-788.

3 Sledz P, Unverdorben P, Beck F, et al. Proc Natl Acad Sci USA 2013; 110:7264-7269.

4 Meyer AS, Gillespie JR, Walther D, et al. Cell 2003; 113:369-381.

5 Itsathitphaisarn O, Wing RA, Eliason WK, et al. Cell 2012; 151:267-277.

6 Schweitzer A, Aufderheide A, Rudack T, et al. Proc Natl Acad Sci USA 2016; 113:78167821.

7 Huang X, Luan B, Wu J, et al. Nat Struct Mol Biol 2016; 23:778-785.

8 Beckwith R, Estrin E, Worden EJ, et al. Nat Struct Mol Biol 2013; 20:1164-1172.

9 Kim YC, Snoberger A, Schupp J, et al. Nat Commun 2015; 6:8520.

10 Stinson BM, Nager AR, Glynn SE, et al. Cell 2013; 153:628-639.

11 Wendler P, Ciniawsky S, Kock M, et al. Biochim Biophys Acta 2012; 1823:2-14. 